\title{
MANN ITERATION CONVERGES FASTER THAN ISHIKAWA ITERATION FOR THE CLASS OF ZAMFIRESCU OPERATORS
}

\author{
G. V. R. BABU AND K. N. V. V. VARA PRASAD \\ Received 3 February 2005; Revised 31 March 2005; Accepted 19 April 2005
}

The purpose of this paper is to show that the Mann iteration converges faster than the Ishikawa iteration for the class of Zamfirescu operators of an arbitrary closed convex subset of a Banach space.

Copyright (c) 2006 Hindawi Publishing Corporation. All rights reserved.

\section{Introduction}

Let $E$ be a normed linear space, $T: E \rightarrow E$ a given operator. Let $x_{0} \in E$ be arbitrary and $\left\{\alpha_{n}\right\} \subset[0,1]$ a sequence of real numbers. The sequence $\left\{x_{n}\right\}_{n=0}^{\infty} \subset E$ defined by

$$
x_{n+1}=\left(1-\alpha_{n}\right) x_{n}+\alpha_{n} T x_{n}, \quad n=0,1,2, \ldots,
$$

is called the Mann iteration or Mann iterative procedure.

Let $y_{0} \in E$ be arbitrary and $\left\{\alpha_{n}\right\}$ and $\left\{\beta_{n}\right\}$ be sequences of real numbers in $[0,1]$. The sequence $\left\{y_{n}\right\}_{n=0}^{\infty} \subset E$ defined by

$$
\begin{aligned}
y_{n+1} & =\left(1-\alpha_{n}\right) y_{n}+\alpha_{n} T z_{n}, \quad n=0,1,2, \ldots, \\
z_{n} & =\left(1-\beta_{n}\right) y_{n}+\beta_{n} T y_{n}, \quad n=0,1,2, \ldots,
\end{aligned}
$$

is called the Ishikawa iteration or Ishikawa iteration procedure.

Zamfirescu proved the following theorem.

Theorem 1.1 [5]. Let $(X, d)$ be a complete metric space, and $T: X \rightarrow X$ a map for which there exist real numbers $a, b$, and $c$ satisfying $0<a<1,0<b, c<1 / 2$ such that for each pair $x$, $y$ in $X$, at least one of the following is true:

$\left(z_{1}\right) d(T x, T y) \leq \operatorname{ad}(x, y)$;

$\left(z_{2}\right) d(T x, T y) \leq b[d(x, T x)+d(y, T y)]$

$\left(z_{3}\right) d(T x, T y) \leq c[d(x, T y)+d(y, T x)]$. 
Then $T$ has a unique fixed point $p$ and the Picard iteration $\left\{x_{n}\right\}_{n=0}^{\infty}$ defined by

$$
x_{n+1}=T x_{n}, \quad n=0,1,2, \ldots,
$$

converges to $p$, for any $x_{0} \in X$.

An operator $T$ which satisfies the contraction conditions $\left(z_{1}\right)-\left(z_{3}\right)$ of Theorem 1.1 will be called a Zamfirescu operator [2].

Definition 1.2 [3]. Let $\left\{a_{n}\right\}_{n=0}^{\infty},\left\{b_{n}\right\}_{n=0}^{\infty}$ be two sequences of real numbers that converge to $a$ and $b$, respectively, and assume that there exists

$$
l=\lim _{n \rightarrow \infty} \frac{\left|a_{n}-a\right|}{\left|b_{n}-b\right|} .
$$

If $l=0$, then we say that $\left\{a_{n}\right\}_{n=0}^{\infty}$ converges faster to $a$ than $\left\{b_{n}\right\}_{n=0}^{\infty}$ to $b$.

Definition 1.3 [3]. Suppose that for two fixed point iteration procedures $\left\{u_{n}\right\}_{n=0}^{\infty}$ and $\left\{v_{n}\right\}_{n=0}^{\infty}$ both converging to the same fixed point $p$ with the error estimates

$$
\begin{aligned}
& \left\|u_{n}-p\right\| \leq a_{n}, \quad n=0,1,2, \ldots, \\
& \left\|v_{n}-p\right\| \leq b_{n}, \quad n=0,1,2, \ldots,
\end{aligned}
$$

where $\left\{a_{n}\right\}_{n=0}^{\infty}$ and $\left\{b_{n}\right\}_{n=0}^{\infty}$ are two sequences of positive numbers (converging to zero). If $\left\{a_{n}\right\}_{n=0}^{\infty}$ converges faster than $\left\{b_{n}\right\}_{n=0}^{\infty}$, then we say that $\left\{u_{n}\right\}_{n=0}^{\infty}$ converges faster than $\left\{v_{n}\right\}_{n=0}^{\infty}$ to $p$.

We use Definition 1.3 to prove our main results.

Based on Definition 1.3, Berinde [3] compared the Picard and Mann iterations of the class of Zamfirescu operators defined on a closed convex subset of a uniformly convex Banach space and concluded that the Picard iteration always converges faster than the Mann iteration, and these were observed empirically on some numerical tests in [1]. In fact, the uniform convexity of the space is not necessary to prove this conclusion, and hence the following theorem [3, Theorem 4] is established in arbitrary Banach spaces.

Theorem 1.4 [3]. Let E be an arbitrary Banach space, $K$ a closed convex subset of E, and $T$ : $K \rightarrow K$ be a Zamfirescu operator. Let $\left\{x_{n}\right\}_{n=0}^{\infty}$ be defined by (1.1) and $x_{0} \in K$, with $\left\{\alpha_{n}\right\} \subset$ $[0,1]$ satisfying

(i) $\alpha_{0}=1$,

(ii) $0 \leq \alpha_{n}<1$ for $n \geq 1$,

(iii) $\sum_{n=0}^{\infty} \alpha_{n}=\infty$.

Then $\left\{x_{n}\right\}_{n=0}^{\infty}$ converges strongly to the fixed point of $T$ and, moreover, the Picard iteration $\left\{x_{n}\right\}_{n=0}^{\infty}$ defined by (1.3) for $x_{0} \in K$, converges faster than the Mann iteration.

Some numerical tests have been performed with the aid of the software package fixed point [1] and raised the following open problem in [3]: for the class of Zamfirescu operators, does the Mann iteration converge faster than the Ishikawa iteration?

The aim of this paper is to answer this open problem affirmatively, that is, to show that the Mann iteration converges faster than the Ishikawa iteration.

For this purpose we use the following theorem of Berinde. 
Theorem 1.5 [2]. Let $E$ be an arbitrary Banach space, $K$ a closed convex subset of $E$, and $T: K \rightarrow K$ be a Zamfirescu operator. Let $\left\{y_{n}\right\}_{n=0}^{\infty}$ be the Ishikawa iteration defined by (1.2) for $y_{0} \in K$, where $\left\{\alpha_{n}\right\}_{n=0}^{\infty}$ and $\left\{\beta_{n}\right\}_{n=0}^{\infty}$ are sequences of real numbers in $[0,1]$ with $\left\{\alpha_{n}\right\}_{n=0}^{\infty}$ satisfying (iii).

Then $\left\{y_{n}\right\}_{n=0}^{\infty}$ converges strongly to the unique fixed point of $T$.

\section{Main result}

Theorem 2.1. Let $E$ be an arbitrary Banach space, $K$ be an arbitrary closed convex subset of $E$, and $T: K \rightarrow K$ be a Zamfirescu operator. Let $\left\{x_{n}\right\}_{n=0}^{\infty}$ be defined by (1.1) for $x_{0} \in K$, and $\left\{y_{n}\right\}_{n=0}^{\infty}$ be defined by (1.2) for $y_{0} \in K$ with $\left\{\alpha_{n}\right\}_{n=0}^{\infty}$ and $\left\{\beta_{n}\right\}_{n=0}^{\infty}$ real sequences satisfying (a) $0 \leq \alpha_{n}, \beta_{n} \leq 1$ and (b) $\sum \alpha_{n}=\infty$. Then $\left\{x_{n}\right\}_{n=0}^{\infty}$ and $\left\{y_{n}\right\}_{n=0}^{\infty}$ converge strongly to the unique fixed point of $T$, and moreover, the Mann iteration converges faster than the Ishikawa iteration to the fixed point of $T$.

Proof. By [2, Theorem 1] (established in [4]), for $x_{0} \in K$, the Mann iteration defined by (1.1) converges strongly to the unique fixed point of $T$.

By Theorem 1.5, for $y_{0} \in K$, the Ishikawa iteration defined by (1.2) converges strongly to the unique fixed point of $T$. By the uniqueness of fixed point for Zamfirescu operators, the Mann and Ishikawa iterations must converge to the same unique fixed point, $p$ (say) of $T$.

Since $T$ is a Zamfirescu operator, it satisfies the inequalities

$$
\begin{aligned}
& \|T x-T y\| \leq \delta\|x-y\|+2 \delta\|x-T x\|, \\
& \|T x-T y\| \leq \delta\|x-y\|+2 \delta\|y-T x\|
\end{aligned}
$$

for all $x, y \in K$, where $\delta=\max \{a, b /(1-b), c /(1-c)\}$, and $0 \leq \delta<1$, see [3].

Suppose that $x_{0} \in K$. Let $\left\{x_{n}\right\}_{n=0}^{\infty}$ be the Mann iteration associated with $T$, and $\left\{\alpha_{n}\right\}_{n=0}^{\infty}$. Now by using Mann iteration (1.1), we have

$$
\left\|x_{n+1}-p\right\| \leq\left(1-\alpha_{n}\right)\left\|x_{n}-p\right\|+\alpha_{n}\left\|T x_{n}-p\right\| .
$$

On using (2.1) with $x=p$ and $y=x_{n}$, we get

$$
\left\|T x_{n}-p\right\| \leq \delta\left\|x_{n}-p\right\|
$$

Therefore from (2.3),

$$
\left\|x_{n+1}-p\right\| \leq\left(1-\alpha_{n}\right)|| x_{n}-p\left\|+\alpha_{n} \delta\right\| x_{n}-p\left\|=\left[1-\alpha_{n}(1-\delta)\right]\right\| x_{n}-p \|
$$

and thus

$$
\left\|x_{n+1}-p\right\| \leq \prod_{k=1}^{n}\left[1-\alpha_{k}(1-\delta)\right] \cdot\left\|x_{1}-p\right\|, \quad n=0,1,2, \ldots
$$

Here we observe that

$$
1-\alpha_{k}(1-\delta)>0 \quad \forall k=0,1,2, \ldots
$$


4 Mann iteration converges faster...

Now let $\left\{y_{n}\right\}_{n=0}^{\infty}$ be the sequence defined by Ishikawa iteration (1.2) for $y_{0} \in K$. Then we have

$$
\left\|y_{n+1}-p\right\| \leq\left(1-\alpha_{n}\right)\left\|y_{n}-p\right\|+\alpha_{n}\left\|T z_{n}-p\right\|
$$

On using (2.2) with $x=p$ and $y=z_{n}$, we have

$$
\left\|T z_{n}-p\right\| \leq \delta\left\|z_{n}-p\right\|+2 \delta\left\|z_{n}-p\right\|=3 \delta\left\|z_{n}-p\right\|
$$

Again using (2.2) with $x=p$ and $y=y_{n}$, we have

$$
\left\|T y_{n}-p\right\| \leq \delta\left\|y_{n}-p\right\|+2 \delta\left\|y_{n}-p\right\|=3 \delta\left\|y_{n}-p\right\|
$$

Now

$$
\left\|z_{n}-p\right\| \leq\left(1-\beta_{n}\right)\left\|y_{n}-p\right\|+\beta_{n}\left\|T y_{n}-p\right\|
$$

and hence by (2.8)-(2.11), we obtain

$$
\begin{aligned}
\left\|y_{n+1}-p\right\| & \leq\left(1-\alpha_{n}\right)\left\|y_{n}-p\right\|+3 \delta \alpha_{n}\left\|z_{n}-p\right\| \\
& \leq\left(1-\alpha_{n}\right)\left\|y_{n}-p\right\|+3 \delta \alpha_{n}\left[\left(1-\beta_{n}\right)\left\|y_{n}-p\right\|+\beta_{n}\left\|T y_{n}-p\right\|\right] \\
& =\left(1-\alpha_{n}\right)\left\|y_{n}-p\right\|+3 \delta \alpha_{n}\left(1-\beta_{n}\right)\left\|y_{n}-p\right\|+3 \delta \alpha_{n} \beta_{n}\left\|T y_{n}-p\right\| \\
& \leq\left(1-\alpha_{n}\right)\left\|y_{n}-p\right\|+3 \delta \alpha_{n}\left(1-\beta_{n}\right)\left\|y_{n}-p\right\|+3 \delta \alpha_{n} \beta_{n} 3 \delta\left\|y_{n}-p\right\| \\
& =\left[\left(1-\alpha_{n}\right)+3 \delta \alpha_{n}\left(1-\beta_{n}\right)+9 \alpha_{n} \beta_{n} \delta^{2}\right] \cdot\left\|y_{n}-p\right\| \\
& =\left[1-\alpha_{n}\left(1-3 \delta+3 \beta_{n} \delta-9 \beta_{n} \delta^{2}\right)\right] \cdot\left\|y_{n}-p\right\| \\
& =\left[1-\alpha_{n}(1-3 \delta)\left(1+3 \beta_{n} \delta\right)\right] \cdot\left\|y_{n}-p\right\| .
\end{aligned}
$$

Here we observe that

$$
1-\alpha_{n}(1-3 \delta)\left(1+3 \beta_{n} \delta\right)>0 \quad \forall k=0,1,2, \ldots
$$

We have the following two cases.

Case (i). Let $\delta \in(0,1 / 3]$. In this case

$$
1-\alpha_{n}(1-3 \delta)\left(1+3 \beta_{n} \delta\right) \leq 1, \quad \forall n=0,1,2, \ldots,
$$

and hence the inequality (2.12) becomes

$$
\left\|y_{n+1}-p\right\| \leq\left\|y_{n}-p\right\| \quad \forall n
$$

and thus,

$$
\left\|y_{n+1}-p\right\| \leq\left\|y_{1}-p\right\| \quad \forall n
$$


We now compare the coefficients of the inequalities (2.6) and (2.16), using Definition 1.3, with

$$
a_{n}=\prod_{k=1}^{n}\left[1-\alpha_{k}(1-\delta)\right], \quad b_{n}=1,
$$

by (b) we have $\lim _{n \rightarrow \infty}\left(a_{n} / b_{n}\right)=0$.

Case (ii). Let $\delta \in(1 / 3,1)$. In this case

$$
1<1-\alpha_{n}(1-3 \delta)\left(1+3 \beta_{n} \delta\right) \leq 1-\alpha_{n}\left(1-9 \delta^{2}\right)
$$

so that the inequality (2.12) becomes

$$
\left\|y_{n+1}-p\right\| \leq\left[1-\alpha_{n}\left(1-9 \delta^{2}\right)\right]\left\|y_{n}-p\right\| \quad \forall n \text {. }
$$

Therefore

$$
\left\|y_{n+1}-p\right\| \leq \prod_{k=1}^{n}\left[1-\alpha_{k}\left(1-9 \delta^{2}\right)\right]\left\|y_{1}-p\right\| .
$$

We compare (2.6) and (2.20), using Definition 1.3 with

$$
a_{n}=\prod_{k=1}^{n}\left[1-\alpha_{k}(1-\delta)\right], \quad b_{n}=\prod_{k=1}^{n}\left[1-\alpha_{k}\left(1-\delta^{2}\right)\right] .
$$

Here $a_{n} \geq 0$ and $b_{n} \geq 0$ for all $n$; and $b_{n} \geq 1$ for all $n$.

Thus $a_{n} / b_{n} \leq a_{n}$ and since $\lim _{n \rightarrow \infty} a_{n}=0$, we have $\lim _{n \rightarrow \infty}\left(a_{n} / b_{n}\right)=0$.

Hence, from Cases (i) and (ii), it follows that $\left\{a_{n}\right\}$ converges faster than $\left\{b_{n}\right\}$, so that the Mann iteration $\left\{x_{n}\right\}$ converges faster than the Ishikawa iteration to the fixed point $p$ of $T$.

COROLlary 2.2. Under the hypotheses of Theorem 2.1, the Picard iteration defined by (1.3) converges faster than the Ishikawa iteration defined by (1.2), to the fixed point of Zamfirescu operator.

Proof. It follows from Theorems 1.4 and 2.1.

Remark 2.3. The Ishikawa iteration (1.2) is depending upon the parameters $\left\{\alpha_{n}\right\}_{n=0}^{\infty}$ and $\left\{\beta_{n}\right\}_{n=0}^{\infty}$ whereas the Mann iteration (1.1) is only on $\left\{\alpha_{n}\right\}_{n=0}^{\infty}$; and by Theorem 2.1, Mann iteration converges faster than the Ishikawa iteration. Now, the Picard iteration (1.3) is free from parameters and Theorem 1.4 says that the Picard iteration converges faster than the Mann iteration.

Perhaps, the reason for this phenomenon is due to increasing the number of parameters in the iteration may increase the damage of the fastness of the convergence of the iteration to the fixed point for the class of Zamfirescu operators. 
6 Mann iteration converges faster...

\section{Acknowledgments}

This work is partially supported by U. G. C. Major Research Project Grant F. 8-8/2003 (SR). One of the authors (G. V. R. Babu) thanks the University Grants commission, India, for the financial support.

\section{References}

[1] V. Berinde, Iterative Approximation of Fixed Points, Editura Efemeride, Baia Mare, 2002.

[2] - On the convergence of the Ishikawa iteration in the class of quasi contractive operators, Acta Mathematica Universitatis Comenianae. New Series 73 (2004), no. 1, 119-126.

[3] _ Picard iteration converges faster than Mann iteration for a class of quasi-contractive operators, Fixed Point Theory and Applications (2004), no. 2, 97-105.

[4] On the convergence of Mann iteration for a class of quasicontractive operators, in preparation, 2004.

[5] T. Zamfirescu, Fix point theorems in metric spaces, Archiv der Mathematik 23 (1992), 292-298.

G. V. R. Babu: Department of Mathematics, Andhra University, Visakhapatnam, Andhra Pradesh, 530003 , India

E-mail address: gvr_babu@hotmail.com

K. N. V. V. Vara Prasad: Department of Mathematics, Dr. L. B. College, Andhra University, Visakhapatnam, Andhra Pradesh, 530 013, India

E-mail address: knvp71@yahoo.co.in 